\title{
Responses to electrical stimulation of denervated human muscle fibres recorded with single fibre EMG
}

\author{
JOŽE TRONTELJ, ERIK STÅLBERG \\ From the Institute of Clinical Neurophysiology, University Medical Centre, Ljubljana, Yugoslavia, and the \\ Department of Clinical Neurophysiology, University Hospital, Uppsala, Sweden
}

SUMMARY Denervated muscle fibres were stimulated electrically with needle electrodes introduced close to a recording single fibre electrode. The denervated muscle fibre could be driven with rates up to $100 \mathrm{~Hz}$. The jitter was large at threshold but low at suprathreshold stimulus strength. There was evidence of discrete low threshold sites along the denervated muscle fibre, seen as stepwise latency change on smoothly changing stimulus strength, ephaptic activation from other fibres and also as extra-discharges originating from such sites.

In this paper we have added new observations which appear to be relevant for the pathophysiology of so called bizarre repetitive discharges, discussed in the succeeding paper. The electrical characteristics of a muscle fibre change with denervation. Hyperexcitability to mechanical and electrical stimulation and even spontaneous activation are typical features of the denervated muscle fibre in situ. In this study some of the characteristics of the denervated muscle have been investigated. Some of the initial results have been reported elsewhere. ${ }^{12}$

\section{Methods and material}

Six patients with a complete cauda equina lesion, brachial plexus lesion or radial nerve lesion of 3 to 11 months duration agreed to participate in the study. The completeness of the lesion was verified by the absence of voluntary activity and of response to electrical nerve stimulation as recorded with surface electrodes. The recordings were made with single fibre electrodes in the tibialis anterior, biceps brachii or extensor digitorum communis muscles. Stimulation was performed intramuscularly with concentric needle electrodes (DISA 13L50) or with a pair of thin needle electrodes insulated almost to the tip, inserted close together, most often at a distance of about $3 \mathrm{~cm}$ from the recording electrode. This was in an attempt to make the stimulation as selective as possible. The stimulation parameters varied for the different experiments. Most often the stimulus strength was less than $50 \mathrm{~V}$ and the frequency between 0.5 and $100 \mathrm{~Hz}$. The depolarisation of denervated muscle

Address for reprint requests: Erik Stålberg, Department of Clinical Neurophysiology, University Hospital, 75014 Uppsala, Sweden.

Received 10 September 1982

Accepted 20 October 1982 fibres by electrical stimulation was quite easily achieved when the stimulus duration was $10 \mathrm{~ms}$ or longer. It was possible however to use much shorter pulses down to $50 \mu \mathrm{s}$ or even less but then the position of the stimulating electrodes became critical. The pulse duration of $50 \mu \mathrm{s}$ or less was preferred in order to minimise the stimulation jitter, that is variability of latency due to the undefined takeoff of the muscle fibre action potential.

\section{Results}

Occasionally a spontaneously fibrillating muscle fibre was found by the stimulating electrode and activated in isolation from other fibres. Then its rhythm was disrupted, introducing a compensatory pause following each stimulus induced response.

On stimulation at high repetition rates, for example, $10-100 \mathrm{~Hz}$ there was a very pronounced progressive increase of the latency, usually associated with a change of the potential shape (decrease of its slopes and amplitude). On double pulse stimulation most fibres were found to have only the subnormal phase of the velocity recovery function, ${ }^{3}$ but some also exhibited the supernormal phase.

When the stimulus strength was near threshold the latency variation in the response was extremely large, sometimes in the range of several thousand $\mu \mathrm{s}$, but when the stimulus was well above threshold the jitter became small, less than $2-5 \mu$ s (fig 1). With increasing stimulus strength new potentials usually entered. Their jitter was large at first but with further increase of stimulus strength it became small. The decrease in jitter was associated with a shortening of latency. Conversely lengthening of latencies and increase in jitter occurred on decreas- 
ing the stimulus strength. Continuous decrease of the jitter and the shortening of the latency when raising the stimulus strength were similar to those occurring in the stimulated nerve fibre, ${ }^{4}$ but were usually more pronounced.

Occasionally a continuous change in stimulus strength, even when well above the threshold, resulted in a sudden stepwise latency change in some muscle fibres. Thus there was an abrupt shortening of the latency of about 1-10 ms on increasing stimulus strength, and conversely, and abrupt restoration to the previous value on decreasing stimulus strength (fig 2a). Similarly, a stepwise latency increase might occur during increasing subnormality when stimulating at high repetition rates (fig $2 b$ ). In both situations, there was an irregular alternation of the two latency values at the transitional stage (fig 2c). Sometimes such a stepwise latency change of a muscle fibre was clearly associated with disappearance or reappearance of responses of another muscle fibre (Stålberg, Trontelj ${ }^{1}$ fig 67).

In multiple spike responses, action potentials from two of the muscle fibres were often linked, that is on threshold stimulation strength they appeared and disappeared together (fig 3a). Sometimes the later spike exhibited a larger jitter and occasionally blocked, indicating threshold activation. Even then the appearance of the late potential depended on the presence of the early potential. When the stimulus was increased the late potential could move closer and its latency could even become shorter than that of the first muscle fibre (fig 3b).

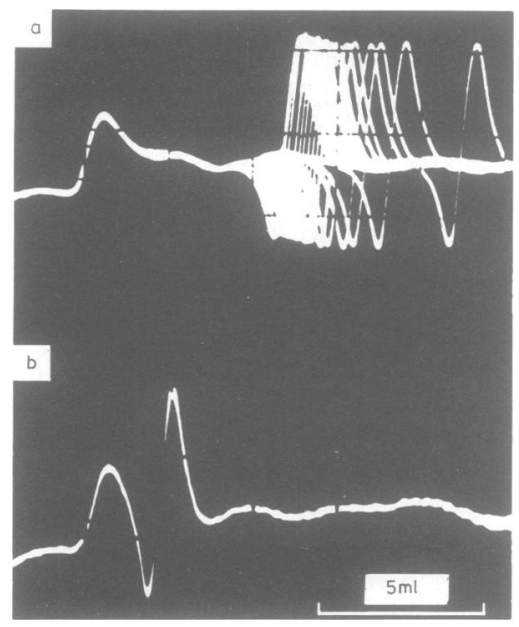

Fig 1 Two denervated muscle fibres responding to electrical stimulation. The jitter of one is large on threshold stimulation (a), but becomes low when stimulus strength is raised to suprathreshold level (b). At the same time the latency decreases. (a)

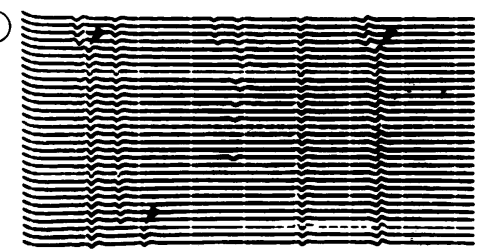

(b)
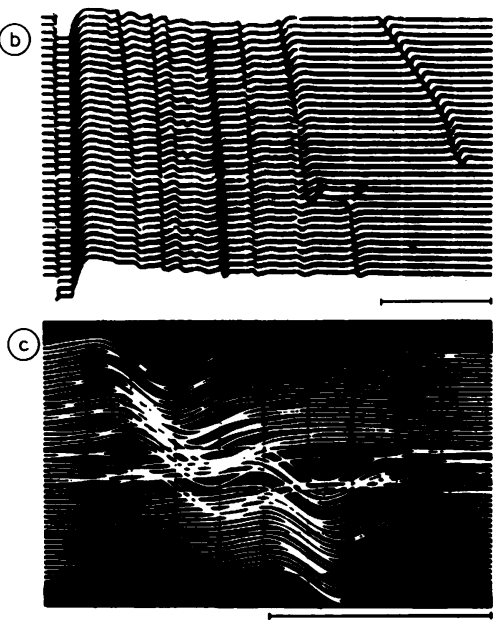

Fig 2 Stepwise prolongation of latencies (arrows in a) on $\sigma \overrightarrow{<}$ smoothly decreasing stimulus strength at repetition rate of 28 응 $H z$. The same phenomenon takes place at constant stimuluso strength but during increasing subnormality at a higher repetition rate, $10 \mathrm{~Hz}(b)$. In both cases there is an uncertainty in the transitional stage (c). This example is taken with decreasing stimulation strength. Stepwise changes of latency can also be associated with appearance or disappearance of responses of another muscle fibre (cf. fig 3).

Many multi-spike recordings revealed another type of late responses, illustrated in fig 4 . These responses evidently belonged to the fibres producing the early response and were considered to be extra-discharges. ${ }^{2}$ This was concluded from the potential shape and evidence of subnormality, that is dependence of action potential amplitude and slopes on the interval to the early responses (fig $4 \mathrm{~b}$ ). The jitter of the extra-discharges was often quite large, for example $10 \mathrm{~ms}$ or more (sometimes even showing a discrete multimodal distribution), but it could be considerably smaller, less than $500 \mu \mathrm{s}$. These responses could follow the early responses every time, or occurred at irregular rates (fig 4c). In order to investigate the site of origin of the extradischarges, double pulse stimulation was used through the same pair of electrodes (fig 5). At an appropriate interstimulus interval, this resulted in extinguishing of the late responses to both of the two stimuli, which could be interpreted to be due to collision. 
(a)

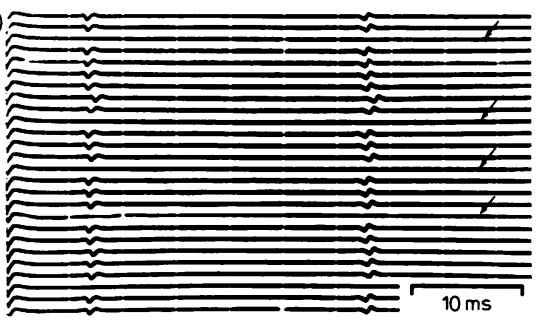

(b)

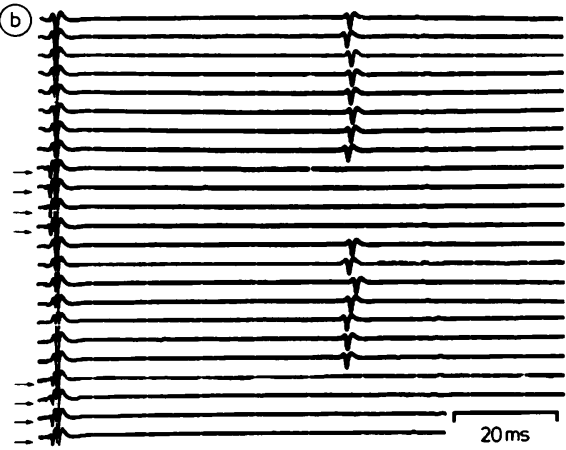

Fig 3 Coupled appearance and disappearance of two muscle fibre action potentials at stimulus strength adjusted to threshold for the first fibre (a). Notice concomitant jitter. (b) Another fibre pair at suprathreshold stimulus strength. A further slight increase in stimulus strength in traces which are marked with arrows resulted in a large latency change of the second fibre's responses which now precedes the first fibre's responses.
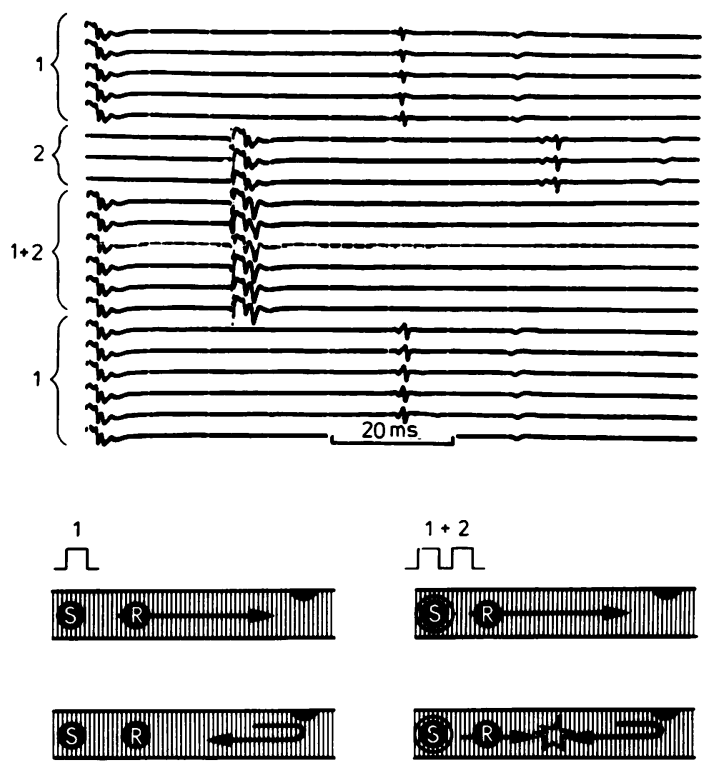

(a)

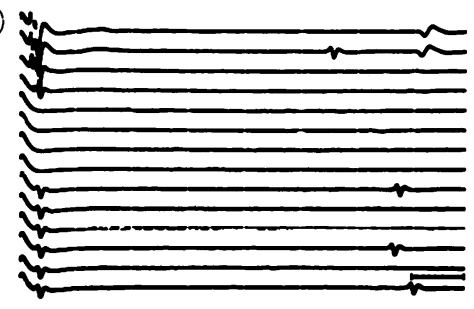

(b)

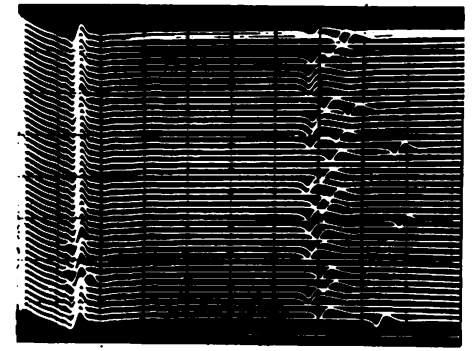

(c)

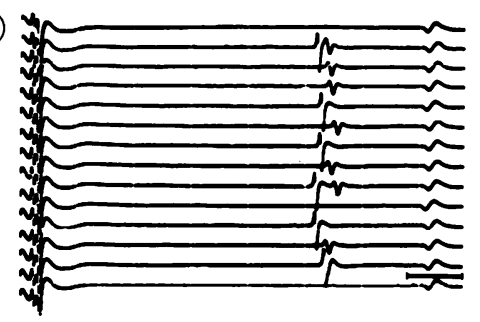

Fig 4 The late potentials are extra-discharges of the muscle fibres producing the early responses. (a) when stimulus strength is below the threshold for the early responses there are no late responses. (b) subnormality of different degrees, depending on the intervals to the late discharges, lowers the amplitude of the action potential and decreases its slopes. (c) the rate of occurrence and the magnitude of the jitter of late discharges varies for different fibres. Time bars $10 \mathrm{~ms}$.

Fig 5 Extra-discharges tested with double pulse stimulation. Either of the two spaced stimuli (indicated as 1 and 2 at the left) elicits the extra-discharges when delivered alone, but extra-discharges to both are extinguished when the stimuli are presented together. This suggests collision, as shown in the schematic drawing. $S=$ stimulating electrode, $\boldsymbol{R}=$ recording electrode. 
A

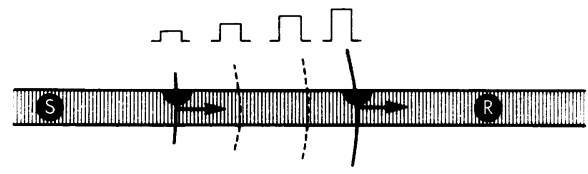

B

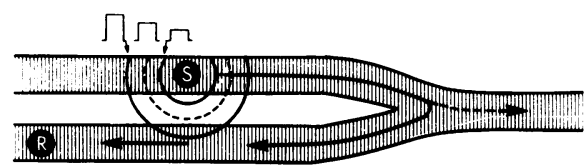

Fig 6 Schematic explanation of the bimodal latency phenomenon as seen in figures 2 and $3 b$. (a) increasing stimulus strength results in movement of the starting point of the action potential to a more distant low threshold site along the muscle fibre. (b) indirect and direct stimulation of a branch of a split muscle fibre with increasing stimulus strength. $S=$ stimulating electrode, $R=$ recording electrode.

\section{Discussion}

The relative ease with which the denervated muscle fibres are activated with electrical stimuli is probably related to their changed resting membrane potential..$^{5-7}$ However, also in the normally innervated muscle, it is not very difficult to stimulate muscle fibres directly (not via their axons), which is in either case recognised from low jitter (less than 2-5 $\mu \mathrm{s}$ ) on suprathreshold stimulus strength. ${ }^{1}$ In contrast to the small jitter on suprathreshold stimulation the jitter is quite large at threshold stimulation strength, considerably larger than at threshold nerve stimulation. This is obviously due to the uncertainty of the point on the membrane from which the depolarisation wave is started, and perhaps even more to the low and slowly rising local potential elicited by the electrical stimulus from which the action potential is triggered. Once the stimulus was well above the threshold, the jitter became very low, less than $5 \mu \mathrm{s}$, in all muscle fibres tested. This indicates normal reliability of propagation of the action potential along the muscle fibre.

The bimodal latency at changing stimulation strength is interpreted as being due to the existence of discrete low threshold sites along the denervated muscle fibres. ${ }^{8}$ When on increasing the stimulus strength the electrical field spreads out it is too weak to start an action potential between two low threshold sites, but when it is increased a little more the starting point of the action potential jumps from the nearer site to the next, more distant one, resulting in stepwise shortening of the latency (fig 6a). Another possible explanation would be the stimula- tion of alternate branches of a split muscle fibre (fig 6B), similar to the axon reflex mechanism. ${ }^{4}$

The phenomenon of coupled potentials from two muscle fibres with different latencies on threshold stimulation, as illustrated in fig 3 , could be interpreted in several ways. One possibility could be that the late responses are in fact extra-discharges, that is repeated discharges of the same muscle fibre such as are illustrated in figs 4 and 5 . This cannot be the case since the different action potential shape and absence of subnormality clearly prove that the late responses were produced by another muscle fibre. Another possibility would be that the late responses are produced by another muscle fibre conducting at a slower velocity, directly activated by the electrical stimulus in the same way as the first muscle fibre. The linked behaviour, even on slight movements of the stimulating electrode, and the fact that the second response was dependent on the presence of the first makes this possibility unlikely. A third possibility would be a recording from two branches of a split muscle fibre. This cannot be true in all cases, as the later responses sometimes showed a larger jitter, associated with occasional blocking. Instead it is assumed that the muscle fibre appearing in the late responses is actually stimulated indirectly, that is by the first fibre's action potential when the latter passes a low threshold site on the second fibre. The considerable scattering of the low threshold sites along the different muscle fibres would account for the large latency difference between the responses support for this explanation is the shortening of the latency brought about by an increase in stimulus strength as illustrated in fig $3 \mathrm{~b}$, when the second fibre presumably becomes activated by the electrical stimulus directly.

Thus the phenomenon of coupled potentials is most likely based on ephaptic impulse transmission between different muscle fibres in the denervated muscle.

Other evidence of such transmission is the stepwise latency change of one muscle fibre associated with appearance and disappearance of responses of another muscle fibre. The newly recruited muscle fibres action potentials must have stimulated the fibre with the bimodal latency phenomenon at one of its low threshold sites closer to the recording electrode than the site of the stimulating electrode. Other evidence of the existence of ephaptic transmission in the denervated muscle includes the paired fibrillations, ${ }^{59}$ and the bizarre repetitive discharges. ${ }^{21011}$ In fact, many characteristics of the electrically elicited responses in denervated muscle closely resemble those of the spontaneous bizarre repetitive discharges. The bizarre repetitive dis- 
charges are based on the low depolarisation threshold of denervated muscle fibres and their ability to be driven at high discharge rates, also demonstrated in the present study.

The so-called extra-discharges are assumed to represent repeated discharges elicited in the same muscle fibre at a low threshold site during a critical period following the passage of the first action potential. The nature of these discharges as well as the antidromic direction of their propagation (with regard to the stimulating electrode) is proved by the collision experiments. This phenomenon is also seen in innervated muscle fibres, for example in myopathies. ${ }^{12}$ Similarly, extra-impulses may be elicited in both motor ${ }^{2}$ and sensory nerve fibres at sites of membrane defects. ${ }^{12}$ The former may give rise to muscle cramp, neuromyotonia or fasciculations, the latter to paraesthesiae or pain. When the threshold at such sites in the muscle fibre becomes further reduced, spontaneous fibrillation potentials are started.

To sum up, most of the phenomena observed in the present study are based on the existence of discrete sites scattered along the denervated muscle fibres where the threshold for spontaneous or externally induced depolarisation is considerably lower than in the segments between.

This work was supported by Research Council of Slovenia, Grant No 90 , by Bob and Vivian Smith Foundation, Houston, Texas (JT) and by the Swedish Medical Research Council Grant No 135 (ES).

\section{References}

${ }^{1}$ Stålberg E, Trontelj JV. Single Fibre Electromyography. The Mirvalle Press, Woking, Surrey, 1979.
${ }^{2}$ Stålberg E, Trontelj JV. Abnormal discharges generated within the motor unit as observed with single fibre electromyography. In: Abnormal Nerves \& Muscles as Impulse Generators, Culp W, Ochoa J, eds. Oxford: Oxford University Press, 1982;443-74.

${ }^{3}$ Stålberg E. Propagation velocity in human muscle fibres in situ. Acta Physiol Scand 1966; 70 Suppl 287,1-112.

4 Stålberg E, Trontelj JV. Demonstration of axon reflexes in human motor nerve fibres. $J$ Neurol Neurosurg Psychiatry 1970;33:571-9.

5 Purves D, Sakmann B. Membrane properties underlying spontaneous activity of denervated muscle fibres. J Physiol (Lond) 1974;239:125.

- Thesleff S, Ward MR. Studies on the mechanism of fibrillation potentials in denervated muscle. $J$ Physiol (Lond) 1975;244:313.

${ }^{7}$ Belmar J. Eyzaguirre C. Pacemaker sites of fibrillation potentials in denervated mammalian muscle. $J$ Neurophysiol 1966;29:425.

${ }^{8}$ Thesleff S. Electrogenic properties of denervated mammalian skeletal muscle. In: Pathogenesis of Human Muscular Dystrophies. Rowland LP, (ed). Amsterdam: Excerpta Medica, 1977;155-60.

9 Harvey AM, Kuffler SW. Synchronisation of spontaneous activity in denervated human muscle. Arch Neurol Psychiat 1943;48:495.

${ }^{10}$ Trontelj JV. Ephaptic transmission in the human skeletal muscle. Zdravstveni Vestnik, 1980;49:181.

11 Trontelj JV, Stålberg E. Bizarre repetitive discharges recorded with single fibre EMG. J Neurol Neurosurg Psychiatry 1983;46:310-6.

${ }^{12}$ Culp W, Ochoa J, Torebjörk E. Ectopic impulse generation in myelinated sensory nerve fibres in man. In: Eds Culp W, Ochoa J Abnormal Nerves \& Muscles as Impulse Generators. Oxford: Oxford University Press, 1982:443-74. 